\title{
Uncovering the Pharmacological of Xiaochaihu Decoction in the Treatment of Pancreatitis Based on the Network Pharmacology
}

\section{Lianghui Zhan}

Tongde Hospital Of Zhejiang Province https://orcid.org/0000-0002-7938-8444

Jinbao Pu

Tongde Hospital Of Zhejiang Province

Yijuan Hu

Tongde Hospital Of Zhejiang Province

Pan Xu

Tongde Hospital Of Zhejiang Province

Weiqing Liang

Tongde Hospital Of Zhejiang Province

Chunlian Ji ( $\boldsymbol{\nabla}$ kjb@zjtongde.com )

Tongde Hospital Of Zhejiang Province

\section{Research}

Keywords: Xiaochaihu Decoction (XD), pancreatitis, network pharmacology

Posted Date: November 12th, 2020

DOI: https://doi.org/10.21203/rs.3.rs-104131/v1

License: (1) This work is licensed under a Creative Commons Attribution 4.0 International License.

Read Full License 


\section{Abstract}

\section{Background}

Xiaochaihu Decoction (XD) has been demonstrated the pharmacodynamic on pancreatitis. This study was aimed to investigate the material and molecular basis of Xiaochaihu Decoction.

\section{Methods}

Firstly, compounds of seven herbs containing XD were collected from TCMSP Database and the putative targets of Pancreatitis were obtained from OMIM, TTD, Genecards Database. Then PPI network was constructed according to the matching results between XD potential targets and pancreatic neoplasms targets. Furthermore, enrichment analysis on GO and KEGG by DAVID utilized bioinformatics resources. Finally, Molecular Docking was performed to simulate the interaction between the active compound of XD and putative targets. In vitro experiment, AR42J cells were induced by LPS and then treated with Quercetin $(25,50,100 \mu \mathrm{M})$. The IL-6, TNF-a, IL-1 $\beta$ levels were detected by Elisa kit and MAPK3, TP53 mRNA expressions were measured by RT-PCR.

\section{Results}

A total of 196 active ingredients and 91 putative targets were selected out. The PPI interaction network analysis demonstrated that Quercetin was the candidate agents and MAPK3, IL-6 and TP53 were the potential targets for the XD treatment of pancreatitis. The KEGG analysis revealed that pathways in cancers, TNF signaling way, MAPK signaling way might play an important role in pancreatitis therapy. And Molecular Docking results showed that Quercetin combined well with MAPK3, IL-6 and TP53. In vitro experiment indicated that, Quercetin inhibited the IL-6, TNF-a, IL-1 $\beta$ levels and MAPK3, TP53 mRNA.

\section{Conclusion}

This study illustrated that Quercetin containing in XD might played an important role in pancreatitis therapy by acting the key genes of MPAK3, IL-6 and TP53 which were associating with inflammation and apoptosis.

\section{Background}

Pancreatitis is highly variable disease characterized by acute inflammation and necrosis of the pancreatic parenchyma which associated with a high mortality of about $20 \%-30 \%$ [1-3]. And it bought on mainly some factors that were gallstones, chronic alcohol and obesity [4-6]. Pancreatitis could divide into local complications including ascites, acute fluid collections, as well as infected necrosis and systemic complications including single organ failure or multiple organ dysfunction syndrome (MODS) $[7,8]$. At present, the major therapeutic measures for pancreatitis was symptomatic treatment such as easement of pain, the correction of fluid, electrolyte, and $\mathrm{pH}$ balances [9]. Currently, there is lack of effective therapeutic strategy for pancreatitis, so valid drug needed to be developed. Due to the 
widespread application of Traditional Chinese Medicine (TCM), it had been testified that Chinese decoctions had significant curative effect on the treatment of Pancreatitis $[10,11]$.

Xiaochaihu Decoction (XD) was chronicle in Shanghan Lun, a famous Chinese ancient book, which was composed of Chaihu (Radix Bupleuri), Banxia (Arum Ternatum Thunb), Renshen (Panax ginseng C. A. Mey.), Gancao (licorice), Huangqin (Scutellariae Radix), Shengjiang (Zingiber officinale Roscoe) and Dazao (Jujubae Fructus), and in recent years experiments showed that XD was beneficial to prevention and cure of Pancreatitis $[12,13]$. Shugen Zhang et al indicated that XD could protect pancreas against chronic injury and improve pancreatic exocrine function in DBTC induced rat CP model [14]. This hinted that XD might be a potential alternative medicine for treatment of Pancreatitis, but its pharmacological mechanism is not well understood.

With the rapid development of Network pharmacology in systems biology, it frequently used in systematically investigate the interaction between Chinese medicine and the complicated human body [15]. Network pharmacology was combined with systems biology, pharmacology and computer technology to explore the complex mechanism by which Chinese formulations treat complex diseases [16]. Furthermore, Network pharmacology applied to the research of TCM could be analysis the rationality of pharmacodynamics mechanism [17].

In the study, we aimed to use a comprehensive network pharmacology-based approach to investigate the mechanisms of how XD exerts the therapeutic effects on Pancreatitis. Further, it was also verified the potential mechanism in vitro experiment.

\section{Materials And Methods}

\section{Data collection of 7 herbs contained in XD}

We collected the chemical ingredients of 7 herbs contained in Xiaohaihu Decoction by Traditional Chinese Medicine Systems Pharmacology Database (TCMSP, https://tcmspw.com/tcmsp.php) [18]. Then screening the requested ingredients according to conditions that oral bioavailability $(\mathrm{OB}) \geq 30 \%$, drug likeness $(D L) \geq 0.18$. The putative targets of 7 herbs contained in Xiaochaihu Decoction were searched in Drugbank (https://www.drugbank.ca/unearth/advanced/bio_entities)

\section{The putative targets of Pancreatitis collection}

By "Pancreatitis" in order to search words, search out the putative targets in GeneCards database (https://auth.lifemapsc.com/) , OMIM database (https://www.omim.org/) and TTD database (http://db.idrblab.net/ttd/) with the with the species limited as "Homo sapiens". Then removed the duplicate value to get the relative putative. 


\section{Gene name correction and common targets screening}

Firstly, Gene name of Xiaochaihu Decoction and Pancreatitis were adjusted by Uniprot database (https://www.uniprot.org/) and then make the intersection map of component targets and disease targets by Venn map to obtain the intersection targets, and further to get the potential therapeutic targets of XD on the treatment of Pancreatitis.

\section{TCM-Compound-Target-Disease Network construction}

Intersection targets obtained from Veen map were reverse screening for corresponding chemical ingredients and herbs. And then TCM-Compound-Target-Disease Network could be constructed which was also visualized by Cytoscape 3.6.1.

\section{Protein protein interaction (PPI) network}

Targets obtained from Venn map was uploaded to STRING Database (http://string-db.org/) and the PPI network was generated with the species limited as "Homo sapiens" and medium confidence as " 0.4 ". And acquired PPI network was imported into Cytoscape 3.6.1 to visually analyze.

\section{Pathway enrichment performance}

The intersection targets were imported into DAVID Database (https://david.ncifcrf.gov/) and then obtained the gene function as well as the effects in the pathway. Gene ontology (GO) and pathway enrichment analyses were performed, setting list type to gene list and limiting species to Homo sapiens, and sorted the top 20 term to draw histogram by Graphpad Prism. Kyoto Encyclopedia of Genes and Genomes database (KEGG, http://www.genome.jp/kegg/) analysis was visualization by ggplot2 database.

\section{Molecular docking method}

Active compounds owed the most targets and targets closely related with Pancreatitis were imported into Discovery Studio 4 software, and then molecular docking was performed by using CDOCKER model.

\section{Experiment validation}

\section{Cell culture}


Rat pancreatic acinar AR42J cells were obtained from China Center for Type Culture Collection (CCTCC, China). AR42J cells were maintained in $1640 \mathrm{RPMI}$ medium containing 10\% FBS, $100 \mathrm{U} / \mathrm{ml}$ penicillin and plated at the density of $1 \times 10^{5}$ cells $/ \mathrm{mL}$ in 96 -well culture plate at $37^{\circ} \mathrm{C}$ and $5 \% \mathrm{CO}_{2}$.

\section{MTT assay}

AR42J cells in each group were treated with LPS (Sigma, America) of various concentrations 1, 2, 4 $\mu \mathrm{g} / \mathrm{mL}$ for 24 hours to investigate the best condition for the subsequent experiment. Quercetin (Sigma, America) at the concentrations of 12.5, 25, 50, $100 \mu \mathrm{M}$ were incubated with AR42J cells for 8 hours after LPS treated. The cells were washed with PBS for 3 times after removing the culture solution and incubated with $5 \mathrm{mg} / \mathrm{mL}$ MTT at $37{ }^{\circ} \mathrm{C}$ for 4 hours. And then $150 \mu \mathrm{L}$ DMSO were added to stop the reaction and the reaction liquid was detected at $570 \mathrm{~nm}$. Each experiment was repeated for 3 times.

\section{The determination of IL-6, TNF- $a$, IL-1 $\beta$}

After treated of LPS and Quercetin, AR42J cells in Cell Culture Dishes of each group were trypsinized and collected by centrifugation at $37^{\circ} \mathrm{C}$ for $5 \mathrm{~min}$ at a speed of $2500 \mathrm{r} / \mathrm{min}$. The factor interleukin- 6 (IL-6), Tumor necrosis factor a (TNF-a) and Interleukin-1 (IL-1 $\beta$ ) were detected by Elisa kit (09/2019, Shanghai Enzyme-linked Biotechnology Co., Ltd) according to the protocol provided by the manufacturer.

\section{TP53 and MAPK3 mRNA expression detected by RT-PCR}

After treated with LPS for 24 hours and Quercetin for another 8 hours, the total RNA of AR42J cells were isolated by Trizol methods (Invitrogen, CA, USA).The integrity were detected by $1 \%$ agarose gel electrophoresis (AGE) and the concertation of total RNA and OD260/OD280 value were measured by micro-spectrophotometer. The procedure of reverse transcription of total RNA was performed in strict accordance with the manufacturer's instructions (CWBIO, Beijing, China). Then the relative expression of TP53 and MAPK3 mRNA were detected by RT-PCR. The PCR primers were designed based on NCBI reference sequence database for each gene and using Primer5 software (Table. 1) and the expansion conditions were as follows: heating to $95^{\circ} \mathrm{C}$ for $10 \mathrm{~min}, 40$ cycles of $5^{\circ} \mathrm{C}$ for $15 \mathrm{sec}, 55^{\circ} \mathrm{C}$ for $30 \mathrm{sec}$ and $72^{\circ} \mathrm{C}$ for $30 \mathrm{sec}$ were applied [19]. GAPDH was used as a reference gene and comparison in expression between groups was made using the $2-\triangle \triangle C T$ method. 
Table.1

PCR primers

\begin{tabular}{|ll|}
\hline Gene & Sequence \\
\hline TP53 & F:5'-TCAGCATCTTATCCGAGTGGAA-3'R:5'-AGGGCACCACCACACTATGTC-3' \\
\hline MAPK3 & F:5'-AACCCAAACAAGCGCATCAC-3'R:5'-TCGGATCGTAGTACTGTTCCAGGTA-3' \\
\hline GAPDH & F:5'-CATGAGAAGTATGACAACAGCCT-3'R:5'-AGTCCTTCCACGATACCAAAGT-3' \\
\hline
\end{tabular}

GADPH was used as an internal control. TP53, tumor protein 53; MAPK3, mitogen-activated protein kinase 3.

\section{Data and statistical analysis}

Statistical analysis was processed with IBM SPSS Statistics 19.0 (SPSS Inc., NY, USA). Data were expressed as the mean \pm SD and analyzed using Student's t-test. Differences between groups were considered to be statistically significant if values of $P<0.05$.

\section{Results}

\section{Active ingredients of Xiaochaihu Decoction}

There were 196 active ingredients of 7 herbs containing in Xiaochaihu Decoction which were collected from TCMSP Database with the limited lists " $\mathrm{OB} \geq 30 \%, \mathrm{OL} \geq 0.18$ ". As is shown in Table.2, the active composition included 17 in Chaihu, 13 in Banxia, 36 in Huangqin, 22 in Renshen, 29 in Daozao, 5 in shenjiang, 94 in Gancao. 
Table.2

Active ingredients information of Xiaochaihu Decoction

\begin{tabular}{|c|c|c|c|c|}
\hline Mol ID & Molecule Name & OB (\%) & DL & Herb \\
\hline MOL000073 & ent-Epicatechin & 48.96 & 0.24 & Scutellariae Radix \\
\hline MOL000096 & (-)-catechin & 49.68 & 0.24 & Jujubae Fructus \\
\hline MOL000098 & quercetin & 46.43 & 0.28 & $\begin{array}{l}\text { Radix Bupleuri] } \\
\text { licorice!Jujubae } \\
\text { Fructus }\end{array}$ \\
\hline MOL000173 & wogonin & 30.68 & 0.23 & Scutellariae Radix \\
\hline MOL000211 & Mairin & 55.38 & 0.78 & $\begin{array}{l}\text { licorice } \text { Jujubaee } \\
\text { Fructus }\end{array}$ \\
\hline MOL000228 & $\begin{array}{l}\text { (2R)-7-hydroxy-5-methoxy-2- } \\
\text { phenylchroman-4-one }\end{array}$ & 55.23 & 0.2 & Scutellariae Radix \\
\hline MOL000239 & Jaranol & 50.83 & 0.29 & licorice \\
\hline MOL000354 & isorhamnetin & 49.6 & 0.31 & $\begin{array}{l}\text { Radix Bupleuri] } \\
\text { licorice }\end{array}$ \\
\hline MOL000358 & beta-sitosterol & 36.91 & 0.75 & $\begin{array}{l}\text { Arum Ternatum } \\
\text { Thunb } \square \text { Panax } \\
\text { ginseng C. A. Mey. } \square \\
\text { Scutellariae Radix } \square \\
\text { Zingiber officinale } \\
\text { Roscoe } \square \text { Jujubae } \\
\text { Fructus }\end{array}$ \\
\hline MOL000359 & sitosterol & 36.91 & 0.75 & $\begin{array}{l}\text { Scutellariae Radix } \\
\text { licorice }\end{array}$ \\
\hline MOL000392 & formononetin & 69.67 & 0.21 & licorice \\
\hline MOL000417 & Calycosin & 47.75 & 0.24 & licorice \\
\hline MOL000422 & kaempferol & 41.88 & 0.24 & $\begin{array}{l}\text { Radix Bupleuri } \\
\text { Panax ginseng C. A. } \\
\text { Mey. .licorice }\end{array}$ \\
\hline MOL000449 & Stigmasterol & 43.83 & 0.76 & $\begin{array}{l}\text { Arum Ternatum } \\
\text { Thunb } \square \text { Panax } \\
\text { ginseng C. A. Mey. } \square \\
\text { Scutellariae Radix } \square \\
\text { Zingiber officinale } \\
\text { Roscoe } \square \text { Jujubae } \\
\text { Fructus } \square \text { Radix } \\
\text { Bupleuri }\end{array}$ \\
\hline MOL000490 & petunidin & 30.05 & 0.31 & Radix Bupleuri \\
\hline MOL000492 & $(+)$-catechin & 54.83 & 0.24 & Jujubae Fructus \\
\hline
\end{tabular}




\begin{tabular}{|c|c|c|c|c|}
\hline Mol ID & Molecule Name & OB (\%) & DL & Herb \\
\hline MOL000497 & licochalcone a & 40.79 & 0.29 & licorice \\
\hline MOL000500 & licochalcone a & 40.79 & 0.29 & licorice \\
\hline MOL000500 & Vestitol & 74.66 & 0.21 & licorice \\
\hline MOL000519 & coniferin & 31.11 & 0.32 & $\begin{array}{l}\text { Arum Ternatum } \\
\text { Thunb }\end{array}$ \\
\hline MOL000525 & Norwogonin & 39.4 & 0.21 & Scutellariae Radix \\
\hline MOL000552 & 5,2'-Dihydroxy-6,7,8-trimethoxyflavone & 31.71 & 0.35 & Scutellariae Radix \\
\hline MOL000627 & Stepholidine & 33.11 & 0.54 & Jujubae Fructus \\
\hline MOL000783 & Protoporphyrin & 30.86 & 0.56 & Jujubae Fructus \\
\hline MOL000787 & Fumarine & 59.26 & 0.83 & $\begin{array}{l}\text { Panax ginseng C. A. } \\
\text { Mey. } \square \text { Jujubae } \\
\text { Fructus }\end{array}$ \\
\hline MOL001454 & berberine & 36.86 & 0.78 & Jujubae Fructus \\
\hline MOL001458 & coptisine & 30.67 & 0.86 & Scutellariae Radix \\
\hline MOL001484 & Inermine & 75.18 & 0.54 & licorice \\
\hline MOL001490 & $\begin{array}{l}\text { bis[(2S)-2-ethylhexyl] benzene-1,2- } \\
\text { dicarboxylate }\end{array}$ & 43.59 & 0.35 & Scutellariae Radix \\
\hline MOL001506 & Supraene & 33.55 & 0.42 & Scutellariae Radix \\
\hline MOL001522 & (S)-Coclaurine & 42.35 & 0.24 & Jujubae Fructus \\
\hline MOL001645 & Linoleyl acetate & 42.1 & 0.2 & Radix Bupleuri \\
\hline MOL001689 & acacetin & 34.97 & 0.24 & Scutellariae Radix \\
\hline MOL001755 & 24-Ethylcholest-4-en-3-one & 36.08 & 0.76 & $\begin{array}{l}\text { Arum Ternatum } \\
\text { Thunb }\end{array}$ \\
\hline MOL001771 & poriferast-5-en-3beta-ol & 36.91 & 0.75 & $\begin{array}{l}\text { Zingiber officinale } \\
\text { Roscoe }\end{array}$ \\
\hline MOL001792 & DFV & 32.76 & 0.18 & licorice \\
\hline MOL002311 & Glycyrol & 90.78 & 0.67 & licorice \\
\hline MOL002565 & Medicarpin & 49.22 & 0.34 & licorice \\
\hline MOL002670 & Cavidine & 35.64 & 0.81 & $\begin{array}{l}\text { Arum Ternatum } \\
\text { Thunb }\end{array}$ \\
\hline
\end{tabular}




\begin{tabular}{|c|c|c|c|c|}
\hline Mol ID & Molecule Name & OB (\%) & DL & Herb \\
\hline MOL002714 & baicalein & 33.52 & 0.21 & $\begin{array}{l}\text { Arum Ternatum } \\
\text { Thunb } \square \text { Scutellariae } \\
\text { Radix }\end{array}$ \\
\hline MOL002773 & beta-carotene & 37.18 & 0.58 & Jujubae Fructus \\
\hline MOL002776 & Baicalin & 40.12 & 0.75 & $\begin{array}{l}\text { Arum Ternatum } \\
\text { Thunb }[\text { Radix } \\
\text { Bupleuri }\end{array}$ \\
\hline MOL002844 & Pinocembrin & 64.72 & 0.18 & licorice \\
\hline MOL002879 & Diop & 43.59 & 0.39 & $\begin{array}{l}\text { Panax ginseng C. A. } \\
\text { Mey. } \square \text { Scutellariae } \\
\text { Radix }\end{array}$ \\
\hline MOL002897 & epiberberine & 43.09 & 0.78 & Scutellariae Radix \\
\hline MOL002908 & 5,8,2'-Trihydroxy-7-methoxyflavone & 37.01 & 0.27 & Scutellariae Radix \\
\hline MOL002909 & 5,7,2,5-tetrahydroxy-8,6-dimethoxyflavone & 33.82 & 0.45 & Scutellariae Radix \\
\hline MOL002910 & Carthamidin & 41.15 & 0.24 & Scutellariae Radix \\
\hline MOL002911 & 2,6,2',4'-tetrahydroxy-6'-methoxychaleone & 69.04 & 0.22 & Scutellariae Radix \\
\hline MOL002913 & Dihydrobaicalin_qt & 40.04 & 0.21 & Scutellariae Radix \\
\hline MOL002914 & Eriodyctiol (flavanone) & 41.35 & 0.24 & Scutellariae Radix \\
\hline MOL002915 & Salvigenin & 49.07 & 0.33 & Scutellariae Radix \\
\hline MOL002917 & 5,2',6'-Trihydroxy-7,8-dimethoxyflavone & 45.05 & 0.33 & Scutellariae Radix \\
\hline MOL002925 & $5,7,2$ ',6'-Tetrahydroxyflavone & 37.01 & 0.24 & Scutellariae Radix \\
\hline MOL002926 & dihydrooroxylin A & 38.72 & 0.23 & Scutellariae Radix \\
\hline MOL002927 & Skullcapflavone II & 69.51 & 0.44 & Scutellariae Radix \\
\hline MOL002928 & oroxylin a & 41.37 & 0.23 & Scutellariae Radix \\
\hline MOL002932 & Panicolin & 76.26 & 0.29 & Scutellariae Radix \\
\hline MOL002933 & 5,7,4'-Trihydroxy-8-methoxyflavone & 36.56 & 0.27 & Scutellariae Radix \\
\hline MOL002934 & NEOBAICALEIN & 104.34 & 0.44 & Scutellariae Radix \\
\hline MOL002937 & DIHYDROOROXYLIN & 66.06 & 0.23 & Scutellariae Radix \\
\hline MOL003410 & Ziziphin_qt & 66.95 & 0.62 & Jujubae Fructus \\
\hline MOL003578 & Cycloartenol & 38.69 & 0.78 & $\begin{array}{l}\text { Arum Ternatum } \\
\text { Thunb }\end{array}$ \\
\hline
\end{tabular}




\begin{tabular}{|c|c|c|c|c|}
\hline Mol ID & Molecule Name & $\mathrm{OB}(\%)$ & DL & Herb \\
\hline MOL003648 & Inermin & 65.83 & 0.54 & $\begin{array}{l}\text { Panax ginseng C. A } \\
\text { Mey. }\end{array}$ \\
\hline MOL003656 & Lupiwighteone & 51.64 & 0.37 & licorice \\
\hline MOL003896 & 7-Methoxy-2-methyl isoflavone & 42.56 & 0.2 & licorice \\
\hline MOL004328 & naringenin & 59.29 & 0.21 & licorice \\
\hline MOL004350 & Ruvoside_qt & 36.12 & 0.76 & Jujubae Fructus \\
\hline MOL004492 & Chrysanthemaxanthin & 38.72 & 0.58 & $\begin{array}{l}\text { Panax ginseng } C . A \\
\text { Mey. }\end{array}$ \\
\hline MOL004598 & $\begin{array}{l}\text { 3,5,6,7-tetramethoxy-2-(3,4,5- } \\
\text { trimethoxyphenyl)chromone }\end{array}$ & 31.97 & 0.59 & Radix Bupleuri \\
\hline MOL004609 & Areapillin & 48.96 & 0.41 & Radix Bupleuri \\
\hline MOL004624 & Longikaurin A & 47.72 & 0.53 & Radix Bupleuri \\
\hline MOL004628 & Octalupine & 47.82 & 0.28 & Radix Bupleuri \\
\hline MOL004644 & Sainfuran & 79.91 & 0.23 & Radix Bupleuri \\
\hline MOL004648 & Troxerutin & 31.6 & 0.28 & Radix Bupleuri \\
\hline MOL004653 & $(+)$-Anomalin & 46.06 & 0.66 & Radix Bupleuri \\
\hline MOL004702 & saikosaponin c_qt & 30.5 & 0.63 & Radix Bupleuri \\
\hline MOL004718 & a-spinasterol & 42.98 & 0.76 & Radix Bupleuri \\
\hline MOL004805 & $\begin{array}{l}\text { (2S)-2-[4-hydroxy-3-(3-methylbut-2- } \\
\text { enyl)phenyl]-8,8-dimethyl-2,3- } \\
\text { dihydropyrano[2,3-f]chromen-4-one }\end{array}$ & 31.79 & 0.72 & licorice \\
\hline MOL004806 & euchrenone & 30.29 & 0.57 & licorice \\
\hline MOL004808 & glyasperin B & 65.22 & 0.44 & licorice \\
\hline MOL004810 & glyasperin F & 75.84 & 0.54 & licorice \\
\hline MOL004811 & Glyasperin C & 45.56 & 0.4 & licorice \\
\hline MOL004814 & Isotrifoliol & 31.94 & 0.42 & licorice \\
\hline MOL004815 & $\begin{array}{l}\text { (E)-1-(2,4-dihydroxyphenyl)-3-(2,2- } \\
\text { dimethylchromen-6-yl)prop-2-en-1-one }\end{array}$ & 39.62 & 0.35 & licorice \\
\hline MOL004820 & kanzonols W & 50.48 & 0.52 & licorice \\
\hline MOL004824 & $\begin{array}{l}\text { (2S)-6-(2,4-dihydroxyphenyl)-2-(2- } \\
\text { hydroxypropan-2-yl)-4-methoxy-2,3- } \\
\text { dihydrofuro[3,2-g]chromen-7-one }\end{array}$ & 60.25 & 0.63 & licorice \\
\hline
\end{tabular}




\begin{tabular}{|c|c|c|c|c|}
\hline Mol ID & Molecule Name & OB (\%) & DL & Herb \\
\hline MOL004827 & Semilicoisoflavone B & 48.78 & 0.55 & licorice \\
\hline MOL004828 & Glepidotin A & 44.72 & 0.35 & licorice \\
\hline MOL004829 & Glepidotin B & 64.46 & 0.34 & licorice \\
\hline MOL004833 & Phaseolinisoflavan & 32.01 & 0.45 & licorice \\
\hline MOL004835 & Glypallichalcone & 61.6 & 0.19 & licorice \\
\hline MOL004838 & $\begin{array}{l}\text { 8-(6-hydroxy-2-benzofuranyl)-2,2-dimethyl- } \\
\text { 5-chromenol }\end{array}$ & 58.44 & 0.38 & licorice \\
\hline MOL004841 & Licochalcone B & 76.76 & 0.19 & licorice \\
\hline MOL004848 & licochalcone G & 49.25 & 0.32 & licorice \\
\hline MOL004849 & $\begin{array}{l}\text { 3-(2,4-dihydroxyphenyl)-8-(1,1-dimethylprop- } \\
\text { 2-enyl)-7-hydroxy-5-methoxy-coumarin }\end{array}$ & 59.62 & 0.43 & licorice \\
\hline MOL004855 & Licoricone & 63.58 & 0.47 & licorice \\
\hline MOL004856 & Gancaonin A & 51.08 & 0.4 & licorice \\
\hline MOL004857 & Gancaonin B & 48.79 & 0.45 & licorice \\
\hline MOL004860 & licorice glycoside E & 32.89 & 0.27 & licorice \\
\hline MOL004863 & $\begin{array}{l}\text { 3-(3,4-dihydroxyphenyl)-5,7-dihydroxy-8-(3- } \\
\text { methylbut-2-enyl)chromone }\end{array}$ & 66.37 & 0.41 & licorice \\
\hline MOL004864 & $\begin{array}{l}\text { 5,7-dihydroxy-3-(4-methoxyphenyl)-8-(3- } \\
\text { methylbut-2-enyl)chromone }\end{array}$ & 30.49 & 0.41 & licorice \\
\hline MOL004866 & $\begin{array}{l}\text { 2-(3,4-dihydroxyphenyl)-5,7-dihydroxy-6-(3- } \\
\text { methylbut-2-enyl)chromone }\end{array}$ & 44.15 & 0.41 & licorice \\
\hline MOL004879 & Glycyrin & 52.61 & 0.47 & licorice \\
\hline MOL004882 & Licocoumarone & 33.21 & 0.36 & licorice \\
\hline MOL004883 & Licoisoflavone & 41.61 & 0.42 & licorice \\
\hline MOL004884 & Licoisoflavone B & 38.93 & 0.55 & licorice \\
\hline MOL004885 & licoisoflavanone & 52.47 & 0.54 & licorice \\
\hline MOL004891 & shinpterocarpin & 80.3 & 0.73 & licorice \\
\hline MOL004898 & $\begin{array}{l}\text { (E)-3-[3,4-dihydroxy-5-(3-methylbut-2- } \\
\text { enyl)phenyl]-1-(2,4-dihydroxyphenyl)prop-2- } \\
\text { en-1-one }\end{array}$ & 46.27 & 0.31 & licorice \\
\hline MOL004903 & liquiritin & 65.69 & 0.74 & licorice \\
\hline
\end{tabular}




\begin{tabular}{|c|c|c|c|c|}
\hline Mol ID & Molecule Name & $\mathrm{OB}(\%)$ & DL & Herb \\
\hline MOL004904 & licopyranocoumarin & 80.36 & 0.65 & licorice \\
\hline MOL004905 & $\begin{array}{l}\text { 3,22-Dihydroxy-11-oxo-delta(12)-oleanene- } \\
\text { 27-alpha-methoxycarbonyl-29-oic acid }\end{array}$ & 34.32 & 0.55 & licorice \\
\hline MOL004907 & Glyzaglabrin & 61.07 & 0.35 & licorice \\
\hline MOL004908 & Glabridin & 53.25 & 0.47 & licorice \\
\hline MOL004910 & Glabranin & 52.9 & 0.31 & licorice \\
\hline MOL004911 & Glabrene & 46.27 & 0.44 & licorice \\
\hline MOL004912 & Glabrone & 52.51 & 0.5 & licorice \\
\hline MOL004913 & $\begin{array}{l}\text { 1,3-dihydroxy-9-methoxy-6-benzofurano[3,2- } \\
\text { c]chromenone }\end{array}$ & 48.14 & 0.43 & licorice \\
\hline MOL004914 & $\begin{array}{l}\text { 1,3-dihydroxy-8,9-dimethoxy-6- } \\
\text { benzofurano[3,2-c]chromenone }\end{array}$ & 62.9 & 0.53 & licorice \\
\hline MOL004915 & Eurycarpin A & 43.28 & 0.37 & licorice \\
\hline MOL004917 & glycyroside & 37.25 & 0.79 & licorice \\
\hline MOL004924 & (-)-Medicocarpin & 40.99 & 0.95 & licorice \\
\hline MOL004935 & Sigmoidin-B & 34.88 & 0.41 & licorice \\
\hline MOL004941 & $\begin{array}{l}\text { (2R)-7-hydroxy-2-(4- } \\
\text { hydroxyphenyl)chroman-4-one }\end{array}$ & 71.12 & 0.18 & licorice \\
\hline MOL004945 & $\begin{array}{l}\text { (2S)-7-hydroxy-2-(4-hydroxyphenyl)-8-(3- } \\
\text { methylbut-2-enyl)chroman-4-one }\end{array}$ & 36.57 & 0.32 & licorice \\
\hline MOL004948 & Isoglycyrol & 44.7 & 0.84 & licorice \\
\hline MOL004949 & Isolicoflavonol & 45.17 & 0.42 & licorice \\
\hline MOL004957 & $\mathrm{HMO}$ & 38.37 & 0.21 & licorice \\
\hline MOL004959 & 1-Methoxyphaseollidin & 69.98 & 0.64 & licorice \\
\hline MOL004961 & Quercetin der. & 46.45 & 0.33 & licorice \\
\hline MOL004966 & 3'-Hydroxy-4'-0-Methylglabridin & 43.71 & 0.57 & licorice \\
\hline MOL004974 & 3'-Methoxyglabridin & 46.16 & 0.57 & licorice \\
\hline MOL004978 & $\begin{array}{l}\text { 2-[(3R)-8,8-dimethyl-3,4-dihydro-2H- } \\
\text { pyrano[6,5-f]chromen-3-yl]-5-methoxyphenol }\end{array}$ & 36.21 & 0.52 & licorice \\
\hline MOL004980 & Inflacoumarin A & 39.71 & 0.33 & licorice \\
\hline MOL004985 & icos-5-enoic acid & 30.7 & 0.2 & licorice \\
\hline
\end{tabular}




\begin{tabular}{|c|c|c|c|c|}
\hline Mol ID & Molecule Name & $\mathrm{OB}(\%)$ & DL & Herb \\
\hline MOL004988 & Kanzonol F & 32.47 & 0.89 & licorice \\
\hline MOL004989 & 6-prenylated eriodictyol & 39.22 & 0.41 & licorice \\
\hline MOL004990 & 7,2',4'-trihydroxy-5-methoxy-3ロarylcoumarin & 83.71 & 0.27 & licorice \\
\hline MOL004991 & 7-Acetoxy-2-methylisoflavone & 38.92 & 0.26 & licorice \\
\hline MOL004993 & 8-prenylated eriodictyol & 53.79 & 0.4 & licorice \\
\hline MOL004996 & gadelaidic acid & 30.7 & 0.2 & licorice \\
\hline MOL005000 & Gancaonin G & 60.44 & 0.39 & licorice \\
\hline MOL005001 & Gancaonin $\mathrm{H}$ & 50.1 & 0.78 & licorice \\
\hline MOL005003 & Licoagrocarpin & 58.81 & 0.58 & licorice \\
\hline MOL005007 & Glyasperins M & 72.67 & 0.59 & licorice \\
\hline MOL005008 & Glycyrrhiza flavonol A & 41.28 & 0.6 & licorice \\
\hline MOL005012 & Licoagroisoflavone & 57.28 & 0.49 & licorice \\
\hline MOL005013 & 18a-hydroxyglycyrrhetic acid & 41.16 & 0.71 & licorice \\
\hline MOL005016 & Odoratin & 49.95 & 0.3 & licorice \\
\hline MOL005017 & Phaseol & 78.77 & 0.58 & licorice \\
\hline MOL005018 & Xambioona & 54.85 & 0.87 & licorice \\
\hline MOL005020 & dehydroglyasperins $\mathrm{C}$ & 53.82 & 0.37 & licorice \\
\hline MOL005030 & gondoic acid & 30.7 & 0.2 & $\begin{array}{l}\text { Arum Ternatum } \\
\text { Thunb }\end{array}$ \\
\hline MOL005308 & Aposiopolamine & 66.65 & 0.22 & $\begin{array}{l}\text { Panax ginseng } C . A \\
\text { Mey. }\end{array}$ \\
\hline MOL005314 & Celabenzine & 101.88 & 0.49 & $\begin{array}{l}\text { Panax ginseng } C \text {. A } \\
\text { Mey. }\end{array}$ \\
\hline MOL005317 & Deoxyharringtonine & 39.27 & 0.81 & $\begin{array}{l}\text { Panax ginseng } C . A \\
\text { Mey. }\end{array}$ \\
\hline MOL005318 & Dianthramine & 40.45 & 0.2 & $\begin{array}{l}\text { Panax ginseng } C . A \\
\text { Mey. }\end{array}$ \\
\hline MOL005320 & arachidonate & 45.57 & 0.2 & $\begin{array}{l}\text { Panax ginseng C. A. } \\
\text { Mey. }\end{array}$ \\
\hline MOL005321 & Frutinone A & 65.9 & 0.34 & $\begin{array}{l}\text { Panax ginseng } C . A \\
\text { Mey. }\end{array}$ \\
\hline
\end{tabular}




\begin{tabular}{|c|c|c|c|c|}
\hline Mol ID & Molecule Name & $\mathrm{OB}(\%)$ & DL & Herb \\
\hline MOL005344 & ginsenoside rh2 & 36.32 & 0.56 & $\begin{array}{l}\text { Panax ginseng C. A. } \\
\text { Mey. }\end{array}$ \\
\hline MOL005348 & Ginsenoside-Rh4_qt & 31.11 & 0.78 & $\begin{array}{l}\text { Panax ginseng } C . A \\
\text { Mey. }\end{array}$ \\
\hline MOL005356 & Girinimbin & 61.22 & 0.31 & $\begin{array}{l}\text { Panax ginseng } C . A \text {. } \\
\text { Mey. }\end{array}$ \\
\hline MOL005357 & Gomisin B & 31.99 & 0.83 & $\begin{array}{l}\text { Panax ginseng C. A. } \\
\text { Mey. }\end{array}$ \\
\hline MOL005360 & malkangunin & 57.71 & 0.63 & $\begin{array}{l}\text { Panax ginseng } C . A \text {. } \\
\text { Mey. }\end{array}$ \\
\hline MOL005360 & malkangunin & 57.71 & 0.63 & Jujubae Fructus \\
\hline MOL005376 & Panaxadiol & 33.09 & 0.79 & $\begin{array}{l}\text { Panax ginseng } C \text {. A. } \\
\text { Mey. }\end{array}$ \\
\hline MOL005384 & suchilactone & 57.52 & 0.56 & $\begin{array}{l}\text { Panax ginseng } C . A \\
\text { Mey. }\end{array}$ \\
\hline MOL005399 & alexandrin_qt & 36.91 & 0.75 & $\begin{array}{l}\text { Panax ginseng } C . A \\
\text { Mey. }\end{array}$ \\
\hline MOL005401 & ginsenoside Rg5_qt & 39.56 & 0.79 & $\begin{array}{l}\text { Panax ginseng C. } A \text {. } \\
\text { Mey. }\end{array}$ \\
\hline MOL006129 & 6-methylgingediacetate2 & 48.73 & 0.32 & $\begin{array}{l}\text { Zingiber officinale } \\
\text { Roscoe }\end{array}$ \\
\hline MOL006936 & 10,13-eicosadienoic & 39.99 & 0.2 & $\begin{array}{l}\text { Arum Ternatum } \\
\text { Thunb }\end{array}$ \\
\hline MOL006937 & $\begin{array}{l}\text { 12,13-epoxy-9-hydroxynonadeca-7,10- } \\
\text { dienoic acid }\end{array}$ & 42.15 & 0.24 & $\begin{array}{l}\text { Arum Ternatum } \\
\text { Thunb }\end{array}$ \\
\hline MOL006957 & $\begin{array}{l}\text { (3S,6S)-3-(benzyl)-6-(4- } \\
\text { hydroxybenzyl)piperazine-2,5-quinone }\end{array}$ & 46.89 & 0.27 & $\begin{array}{l}\text { Arum Ternatum } \\
\text { Thunb }\end{array}$ \\
\hline MOL006967 & beta-D-Ribofuranoside, xanthine- 9 & 44.72 & 0.21 & $\begin{array}{l}\text { Arum Ternatum } \\
\text { Thunb }\end{array}$ \\
\hline MOL007213 & Nuciferin & 34.43 & 0.4 & Jujubae Fructus \\
\hline MOL008034 & $21302-79-4$ & 73.52 & 0.77 & Jujubae Fructus \\
\hline MOL008206 & Moslosooflavone & 44.09 & 0.25 & Scutellariae Radix \\
\hline MOL008647 & Moupinamide & 86.71 & 0.26 & Jujubae Fructus \\
\hline MOL008698 & Dihydrocapsaicin & 47.07 & 0.19 & $\begin{array}{l}\text { Zingiber officinale } \\
\text { Roscoe }\end{array}$ \\
\hline MOL010415 & 11,13-Eicosadienoic acid, methyl ester & 39.28 & 0.23 & Scutellariae Radix \\
\hline
\end{tabular}




\begin{tabular}{|c|c|c|c|c|}
\hline Mol ID & Molecule Name & OB (\%) & DL & Herb \\
\hline MOL012245 & 5,7,4'-trihydroxy-6-methoxyflavanone & 36.63 & 0.27 & Scutellariae Radix \\
\hline MOL012246 & 5,7,4'-trihydroxy-8-methoxyflavanone & 74.24 & 0.26 & Scutellariae Radix \\
\hline MOL012266 & rivularin & 37.94 & 0.37 & Scutellariae Radix \\
\hline MOL012921 & stepharine & 31.55 & 0.33 & Jujubae Fructus \\
\hline MOL012940 & Spiradine A & 113.52 & 0.61 & Jujubae Fructus \\
\hline MOL012946 & zizyphus saponin I_qt & 32.69 & 0.62 & Jujubae Fructus \\
\hline MOL012961 & jujuboside A_qt & 36.67 & 0.62 & Jujubae Fructus \\
\hline MOL012976 & coumestrol & 32.49 & 0.34 & Jujubae Fructus \\
\hline MOL012980 & Daechuine S6 & 46.48 & 0.79 & Jujubae Fructus \\
\hline MOL012981 & Daechuine S7 & 44.82 & 0.83 & Jujubae Fructus \\
\hline MOL012986 & Jujubasaponin V_qt & 36.99 & 0.63 & Jujubae Fructus \\
\hline MOL012989 & Jujuboside C_qt & 40.26 & 0.62 & Jujubae Fructus \\
\hline MOL012992 & Mauritine D & 89.13 & 0.45 & Jujubae Fructus \\
\hline MOL013187 & Cubebin & 57.13 & 0.64 & Radix Bupleuri \\
\hline MOL013357 & $\begin{array}{l}\text { (3S,6R,8S,9S,10R,13R,14S,17R)-17- } \\
{[(1 \mathrm{R}, 4 \mathrm{R})-4-\mathrm{ethy}-1,5-\text { dimethylhexyl]-10,13- }} \\
\text { dimethyl-2,3,6,7,8,9,11,12,14,15,16,17- } \\
\text { dodecahydro-1H- } \\
\text { cyclopenta[a]phenanthrene-3,6-diol }\end{array}$ & 34.37 & 0.78 & Jujubae Fructus \\
\hline
\end{tabular}

\section{Intersection targets of Xiaochaihu Decoction and Pancreatitis}

Xiaochaihu Decoction had 169 targets obtained from Drugbank Database and Pancreatitis had 2344 targets collected from OMIM, GeneCards and TTD Databases. Then, Venn map showed that it had 91 intersection targets on Xiaochaihu Decoction and Pancreatitis (Figure.1).

\section{Herb-Compound-Target-Disease Network analysis}

To clarify the relationship between herb of Xiaochaihu Decoction, compound target and pancreatitis targets, the herb-compound-target-disease network was constructed by Cytoscape software. The network had 293 nodes consisted of 1 disease, 7 herbs, 194 compounds and 91 putative targets. In the terms of 
the relationship between compounds and targets, there were 12 compounds having targets greater than 30, and they were MOL000098 (Quercetin), MOL000422 (Kaempferol), MOL003896 (7-Methoxy-2-methyl isoflavone), MOL000787 (Fumarine), MOL000354 (Isorhamnetin), MOL000392 (formononetin), MOL002565 (Medicarpin), MOL000358 (Beta-sitosterol), MOL000449 (Stigmasterol), MOL004978(2[(3R)-8,8-dimethyl-3,4-dihydro-2H-pyrano[6,5-f]chromen-3-yl]-5-methoxyphenol), MOL000500 (Vestitol), MOL004891 (Shinpterocarpin) acting on 75, 44, 43, 38, 34, 34, 34, 32, 31, 31, 30, 30 targets respectively. And PTGS2, HSP90A, CAMKK2, ESR1, AR, PTGS1, NOS2, NCOA2, PRSS1, F10 and SCN5A were interact with $138,100,97,90,89,86,83,74,73$ and 71 compounds (Figure.2).

\section{PPI interaction network analysis}

The common targets of compounds and pancreatitis were putted into STRING database to obtain the PPI interaction network in order to provide an intuitive understand of the mechanism of Xiaochaihu Decoction acting on pancreatitis. By the analysis of STRING, it was showed that the network was composed with 91 nodes as well as 1015 edges and the average node degree was 22.3. Furthermore, network topological analysis indicated that the top 3 degrees were Mitogen-activated protein kinase 3 (MAPK3, degree=58), Interleukin - 6 (IL-6, degree=57), Tumor Protein (TP53, degree=56) which involving with the cell growth, cell apoptosis as well as inflammatory response, had the greater node degree value than other targets Figure.3.

\section{Enrichment of functions analysis}

To clarified the mechanism of Xiaochaihu Decoction on pancreatitis in detail, the Go enrichment analysis was performed by DAVID Bioinformatics Resources. Go enrichment analysis got 484 items including 363 items of Biological process (BP), 53 items of Cell Component (CC) and 68 items of Molecular Function (MF) $(P<0.05)$ and the items which Gene count proportion greater than 10 were showed as Figure.4. Biological process was mainly related to positive regulation of transcription from RNA polymerase II promoter and Molecular Function was closely in connection with protein binding.

KEGG pathway analysis indicated that Xiaochaihu Decoction exerted its pharmacological effects on pancreatitis was closely associated with pathways in cancers (fold enrichment $=5.4, P<0.001$ ), TNF signaling way (fold enrichment=12.2, $P<0.001$ ), PI3K-Akt signaling pathway (fold enrichment=3.7, $P<0.001$ ), Influenza A (fold enrichment=7.1, $P<0.001$ ), Chagas disease (fold enrichment=11.0, $P<0.001$ ) and MAPK signaling pathway (fold enrichment $=4.5, P<0.001$ ) which were involving with MAPK3, TP53, TNF and so on (Figure.5).

\section{Molecular docking to determine the potential targets}


Three targets (IL-6, MAPK3, TP53) were selected according to the results of PPI network as the core targets of Xiaochaihu Decoction treated pancreatitis. And Herb-compound-Target-Disease network indicated Quercetin could not only interacted with 75 targets but also closely related with the three targets. Therefore, Molecular Docking technology was used to simulate the interaction between Quercetin and the three targets. The results showed that Quercetin interacted with the IL-6, MAPK3, TP53, forming Vander Waals, Carbon hydrogen bond, Pi-sulfur, Salt Bridge and so on (Figure.6).

\section{The viability of LPS treated AR42J cells for various dose}

To investigate the best concentrations of LPS to build pancreatitis model in vitro, AR42J cells viability were measured following treatment of $1,2,4 \mu \mathrm{g} / \mathrm{mL}$ LPS for 24 hours. As is shown in Figure.7 A, AR42J cells viability were achieve to nearly $60 \%$ with the treatment of LPS at the dose of $2 \mathrm{mg} / \mathrm{mL}$ for 24 hours which was for the subsequent experiment.

According to the results of Herb-Compound-Target-Disease Network analysis, Quercetin was the mainly active compound of Xiaochaihu Decoction. Quercetin $(12.5,25,50,100 \mu \mathrm{M})$ were treated with AR42J cells for 8 hours after AR42J cells were incubated with LPS $(2 \mathrm{mg} / \mathrm{mL})$ for 24 hours to detect cell viability the by MTT assay. It was showed that Quercetin at the concentrations of 25,50 and $100 \mu \mathrm{M}$ significantly improved the cells viability $(P<0.05,0.01)$, but Quercetin at dose the of $12.5 \mu \mathrm{M}$ had less effect on it (Figure.7 B).

\section{Quercetin inhibited the productions of IL-6, IL-1 $\beta$ and TNF- $a$}

According to the PPI interaction network analysis, Xiaochaihu Decoction was closely association with inflammatory reaction. Therefore, the levels of IL-6, IL-1 $\beta$ and TNF- $\alpha$ in LPS-induced pancreatitis model in vitro were detected. LPS significantly increased the secretion of IL-1 $\beta$, IL- 6 and TNF- $a$ compared with control group. At expected, Quercetin at 25, 50,100 $\mu \mathrm{M}$ were all reduced the generations of these inflammatory factors $(P<0.05,0.01$, Figure.8 $\mathrm{A}, \mathrm{B}$ and $\mathrm{C})$.

\section{Quercetin downregulated the relative expression of MAPK3 and TP53}

The PPI network and KEGG results all showed that Xiaochaihu Decoction functioning in the treatment of insomnia were involving with MAPK3 and TP53 which were further confirmed by Molecular docking technology. In the vitro experiment (Figure.9 A and B), MAPK3 and TP53 were both upregulated after AR42J cells treated with LPS, significantly $(P<0.01)$. However, compared with LPS group, Quercetin obviously reduced the relative expressions MAPK3 and TP53 $(P<0.05,0.01)$.

\section{Discussion}


Xiaochaihu Decoction was an ancient herbal formula which had been as the therapeutic agents for the treatment of pancreatitis in clinical [20]. In this study, a network pharmacology approach was applied to construct the "Herb-Compound-Target-Disease Network" in order to explore the underlying mechanism of Xichaihu Decoction in pancreatitis. Results of the study described herein revealed that Xiaochaihu Decoction exerted on the pancreatitis by multiple pathways, target points.

According to the results of Network pharmacology analysis, Quercetin ( $O B=46.43 \%, D L=0.28)$, Kaempferol $(\mathrm{OB}=41.88 \%, \mathrm{DL}=0.24)$ and so on became the potential bioactive compounds. Quercetin is a flavonoid found which could attenuated pancreatic and ileal pathological damages through reduced the production of IL-6, TNF-a, IL-1 $\beta$ [21]. Besides, In vitro active experiment shows that, Quercetin had a significant anti-pancreatitis activity through reducing the intracellular ROS production and enhancing apoptotic cell death[22]. Kaempferol, a kind of polyphenol, ameliorated acute inflammatory and nociceptive symptoms in pancreatitis [23]. Yun Jung Lee et al had been demonstrated that kaempferol could protect pancreatic cells from dRib-induced associated oxidative damage by inhibiting the intracellular ROS and apoptosis [24]. And the study also indicated that Quercetin at the concentrations of 25,50 and $100 \mu \mathrm{M}$ indeed improved the viability of AR42J cells which was triggered inflammatory damage by LPS.

KEGG analysis revealed that pathways in cancers, TNF signaling way, PI3K-Akt signaling pathway and MAPK signaling pathway had linkage with Xiaochaihu Decoction treatment for pancreatitis. As we all known, TNF was mainly generated by activated mononuclear macrophages and involved in inducing the production of Inflammatory cytokines such as IL-6 causing the necrosis of pancreatic tissue [25]. Thus, TNF signaling way might be the important proinflammatory cytokine for the occurrence and development of pancreatitis. The productions of TNF- $a, \mathrm{IL}-6$ and IL-1 $\beta$ in the AR42J cells were decreased with the treated with Quercetin at the concentrations of 25, 50 and $100 \mu \mathrm{M}$ which demonstrated Xiaochaihu Decoction as a treatment for pancreatitis via inhibiting the generations of inflammation factors.

PPI network analysis also showed that the degree of MAPK3 (degree = 58), IL-6 (degree = 57), TP53 (degree $=56$ ) were higher than others. MAPK was an important transmitter of signals from the cell surface to the inside of the nucleus and MAPK 3 acted as an essential component of the MAPK signal transduction pathway [26]. The MAPK cascade mediates diverse biological functions such as cell growth, adhesion, survival and differentiation through the regulation of transcription, translation, cytoskeletal rearrangements [27]. It had been reported that pancreatitis could be alleviated via reducing the production of TNF-a, IL-6, IL-1 $\beta$ which was associated with regulating the MAPK signaling way [28]. Xiang-Peng Zeng et al found that inhibiting the MAPK cascade including MAPK3 might be a potential anti-inflammatory strategy for pancreatitis [29].

IL-6 was one of the most biologically active cytokines, had many biological functions and was considered as the reliable marker for severity in acute pancreatitis [30]. IL-6 promoted the neutrophil function p-regulation, cytokine section and inflammatory mediator production leading the development of 
pancreatitis [31]. Besides, IL-6 triggered the strong STAT3 phosphorylation in the pancreas and high circulating levels of neutrophil attractant CXCL1 which resulting in the severity of pancreatitis [32].

TP53, the regulatory factor of apoptosis, could interact with $\mathrm{Bcl}_{2}$ family proteins in the cytoplasm that causing mitochondrial outer membrane permeability increased and cell apoptosis [33]. In clinical, pancreatic tissues from patients with pancreatitis exhibited apoptotic nuclei, and increased p53 expression [34]. Consistently, Lei Zhou et al found that TP53 suppressed on mouse pancreatitis model obviously inhibited pancreatic acinar cell apoptosis and the inflammation [35].

In this study, inflammation factors and apoptosis factors (MAPK3, IL-6 and TP53) were significantly downregulated by Quercetin. Given that inflammation and apoptosis were closely related with pancreatitis, they were likely to be the molecules regulated by Xiaochaihu Decoction in the treatment of pancreatitis.Molecular docking was the widely use technology for calculating protein-ligand interactions [36]. And the results also showed that Quercetin could docked well with MAPK3, IL-6 and TP53. In other words, Xiaochaihu Decoction exerted pharmacodynamic actions on pancreatitis via the interaction between active ingredients and key proteins.

\section{Conclusion}

In general, this study combined network pharmacology method and molecular docking technology to demonstrate the potential mechanism of Xiaochaihu Decoction treated with pancreatitis that Quercetin might played an important role in pancreatitis therapy by acting the key genes of MPAK3, IL-6 and TP53. Furthermore, it was demonstrated that Quercetin as the mainly active compound of Xiaochaihu Decoction reduced the inflammation and apoptosis factors (IL-1 $\beta$, IL-6, TNF-a, MAPK3, TP53) generation to in vitro experiment. However, the further mechanism about Xiaochaihu Decoction treated on insomnia was still remined to be systematic explored.

\section{Abbreviations}

$\mathrm{XD}=$ Xiaochaihu Decoction

$\mathrm{TCM}=$ Traditional Chinese Medicine

TCMSP = Traditional Chinese Medicine Systems Pharmacology

$\mathrm{BP}=$ Biological process

$\mathrm{CC}=$ Cell Component

MF = Molecular Function

$\mathrm{GO}=$ Gene ontology 
KEGG = Kyoto Encyclopedia of Genes and Genomes

IL-1 $\beta=$ Interleukin $-1 \beta$

IL-6 = factor interleukin-6

TNF-a $=$ Tumor necrosis factor $a$

\section{Declarations}

\section{Acknowledgments}

The presentation of the manuscript had been preprinted before.

\section{Author's contribution}

Lianghui Zhan and Chunlian Ji conceived and designed the experiments. Lianghui Zhan and Jinbao Pu contributed significantly to analysis and manuscript preparation. Yijuan Hu, Pan Xu and Weiqing Liang helped perform the analysis with constructive discussions.

\section{Funding}

No

\section{Availability of data and materials}

All data generated or analyzed during this study are included in this published article. Please contact the author for the code of the software and the documentation.

\section{Ethics approval and consent to participate}

\section{Consent for publication}

Not applicable.

\section{Conflict of interest}

The authors declare no conflicts of interest in association with this manuscript. 


\section{References}

1. Bhatia M, Wong FL, Cao Y, Lau HY, Huang J, Puneet P, Chevali L: Pathophysiology of acute pancreatitis. Pancreatology 2005, 5(2-3):132-144.

2. Saloman JL, Albers KM, Cruz-Monserrate Z, Davis BM, Edderkaoui M, Eibl G, Epouhe AY, Gedeon JY, Gorelick FS, Grippo PJ et al: Animal Models: Challenges and Opportunities to Determine Optimal Experimental Models of Pancreatitis and Pancreatic Cancer. Pancreas 2019, 48(6):759-779.

3. Shi C, Hou C, Zhu X, Huang D, Peng Y, Tu M, Li Q, Miao Y: SRT1720 ameliorates sodium taurocholateinduced severe acute pancreatitis in rats by suppressing NF-kappaB signalling. Biomed Pharmacother 2018, 108:50-57.

4. Mcllwrath SL, Westlund KN: Pharmacological attenuation of chronic alcoholic pancreatitis induced hypersensitivity in rats. World J Gastroenterol 2015, 21(3):836-853.

5. Khatua B, El-Kurdi B, Singh VP: Obesity and pancreatitis. Curr Opin Gastroentero/ 2017, 33(5):374382.

6. McPherson SJ, O'Reilly DA, Sinclair MT, Smith N: The use of imaging in acute pancreatitis in United Kingdom hospitals: findings from a national quality of care study. Br J Radio/2017, 90(1080):20170224.

7. Banks PA, Freeman ML, Practice Parameters Committee of the American College of G: Practice guidelines in acute pancreatitis. Am J Gastroentero/ 2006, 101(10):2379-2400.

8. Nesvaderani M, Eslick GD, Vagg D, Faraj S, Cox MR: Epidemiology, aetiology and outcomes of acute pancreatitis: A retrospective cohort study. Int J Surg 2015, 23(Pt A):68-74.

9. Rijkers AP, van Eijck CH: Acute Pancreatitis. N Engl J Med 2017, 376(6):596-597.

10. Li J, Zhang S, Zhou R, Zhang J, Li ZF: Perspectives of traditional Chinese medicine in pancreas protection for acute pancreatitis. World J Gastroenterol 2017, 23(20):3615-3623.

11. Zhu L, Li JY, Zhang YM, Kang HX, Chen H, Su H, Li J, Tang WF: Pharmacokinetics and pharmacodynamics of Shengjiang decoction in rats with acute pancreatitis for protecting against multiple organ injury. World J Gastroentero/ 2017, 23(46):8169-8181.

12. Hu Q, Wei S, Wen J, Zhang W, Jiang Y, Qu C, Xiang J, Zhao Y, Peng X, Ma X: Network pharmacology reveals the multiple mechanisms of Xiaochaihu decoction in the treatment of non-alcoholic fatty liver disease. BioData Min 2020, 13:11.

13. Su SB, Li YQ, Shen HY, Motoo Y: [Effects of Chinese herbal medicines on spontaneous chronic pancreatitis in rats and the pathological relationships between formulas and syndromes]. Zhong $X i$ Yi Jie He Xue Bao 2006, 4(4):358-362.

14. Zhang SK, Cui NQ, Zhuo YZ, Li DH, Liu JH: Modified Xiaochaihu Decoction 0 prevents the progression of chronic pancreatitis in rats possibly by inhibiting transforming growth factorbeta1/Sma- and mad-related proteins signaling pathway. Chin J Integr Med 2013, 19(12):935-939.

15. Liu JF, Hu AN, Zan JF, Wang P, You QY, Tan AH: Network Pharmacology Deciphering Mechanisms of Volatiles of Wendan Granule for the Treatment of Alzheimer's Disease. Evid Based Complement 
Alternat Med 2019, 2019:7826769.

16. Zeng L, Yang K, Liu H, Zhang G: A network pharmacology approach to investigate the pharmacological effects of Guizhi Fuling Wan on uterine fibroids. Exp Ther Med 2017, 14(5):46974710.

17. Yu G, Wang W, Wang X, Xu M, Zhang L, Ding L, Guo R, Shi Y: Network pharmacology-based strategy to investigate pharmacological mechanisms of Zuojinwan for treatment of gastritis. $B M C$ Complement Altern Med 2018, 18(1):292.

18. Wang CH, Zhong Y, Zhang Y, Liu JP, Wang YF, Jia WN, Wang GC, Li Z, Zhu Y, Gao XM: A network analysis of the Chinese medicine Lianhua-Qingwen formula to identify its main effective components. Mol Biosyst 2016, 12(2):606-613.

19. Hu Z, Zhang X, Yang H, Qin S, Liu Y, Xiong W, Yuan B, Li L, Yao W, Wu D: Alterations in expression levels of genes in p53-related pathways determined using RNA-Seq analysis in patients with breast cancer following CIK therapy. Oncol Lett 2017, 14(6):7917-7922.

20. Li SM, Xu SJ, Li SM, Hong WX: [Study on clinical experience of using xiaochaihu decoction]. Zhongguo Zhong Xi Yi Jie He Za Zhi 2014, 34(10):1264-1266.

21. Junyuan Z, Hui X, Chunlan H, Junjie F, Qixiang M, Yingying L, Lihong L, Xingpeng W, Yue Z: Quercetin protects against intestinal barrier disruption and inflammation in acute necrotizing pancreatitis through TLR4/MyD88/p38MAPK and ERS inhibition. Pancreatology 2018, 18(7):742-752.

22. Seo JY, Pandey RP, Lee J, Sohng JK, Namkung W, Park YI: Quercetin 3-0-xyloside ameliorates acute pancreatitis in vitro via the reduction of ER stress and enhancement of apoptosis. Phytomedicine 2019, 55:40-49.

23. Kim SH, Park JG, Sung GH, Yang S, Yang WS, Kim E, Kim JH, Ha VT, Kim HG, Yi YS et al: Kaempferol, a dietary flavonoid, ameliorates acute inflammatory and nociceptive symptoms in gastritis, pancreatitis, and abdominal pain. Mol Nutr Food Res 2015, 59(7):1400-1405.

24. Lee YJ, Suh KS, Choi MC, Chon S, Oh S, Woo JT, Kim SW, Kim JW, Kim YS: Kaempferol protects HITT15 pancreatic beta cells from 2-deoxy-D-ribose-induced oxidative damage. Phytother Res 2010, 24(3):419-423.

25. Scotece M, Conde J, Abella V, Lopez V, Francisco V, Ruiz C, Campos V, Lago F, Gomez R, Pino J et al: Oleocanthal Inhibits Catabolic and Inflammatory Mediators in LPS-Activated Human Primary Osteoarthritis (OA) Chondrocytes Through MAPKs/NF-kappaB Pathways. Cell Physiol Biochem 2018, 49(6):2414-2426.

26. Drosten M, Barbacid M: Targeting the MAPK Pathway in KRAS-Driven Tumors. Cancer Cell 2020, 37(4):543-550.

27. Cao MH, Xu J, Cai HD, Lv ZW, Feng YJ, Li K, Chen CQ, Li YY: p38 MAPK inhibition alleviates experimental acute pancreatitis in mice. Hepatobiliary Pancreat Dis Int 2015, 14(1):101-106.

28. Zhang Z, Liu Q, Zang H, Shao Q, Sun T: Oxymatrine protects against l-arginine-induced acute pancreatitis and intestine injury involving Th1/Th17 cytokines and MAPK/NF-kappaB signalling. Pharm Biol 2019, 57(1):595-603. 
29. Zeng XP, Wang LJ, Guo HL, He L, Bi YW, Xu ZL, Li ZS, Hu LH: Dasatinib ameliorates chronic pancreatitis induced by caerulein via anti-fibrotic and anti-inflammatory mechanism. Pharmacol Res 2019, 147:104357.

30. Perez S, Rius-Perez S, Finamor I, Marti-Andres P, Prieto I, Garcia R, Monsalve M, Sastre J: Obesity causes PGC-1alpha deficiency in the pancreas leading to marked IL-6 upregulation via NF-kappaB in acute pancreatitis. J Pathol 2019, 247(1):48-59.

31. Wang $\mathrm{Y}, \mathrm{Bu} \mathrm{C}$, Wu K, Wang $\mathrm{R}$, Wang J: Curcumin protects the pancreas from acute pancreatitis via the mitogenactivated protein kinase signaling pathway. Mol Med Rep 2019, 20(4):3027-3034.

32. Zhang H, Neuhofer P, Song L, Rabe B, Lesina M, Kurkowski MU, Treiber M, Wartmann T, Regner S, Thorlacius $\mathrm{H}$ et al: IL-6 trans-signaling promotes pancreatitis-associated lung injury and lethality. $J$ Clin Invest 2013, 123(3):1019-1031.

33. Kamp WM, Wang PY, Hwang PM: TP53 mutation, mitochondria and cancer. Curr Opin Genet Dev 2016, 38:16-22.

34. Singh L, Bakshi DK, Majumdar S, Arora SK, Vasishta RK, Wig JD: Mitochondrial dysfunction and apoptosis of acinar cells in chronic pancreatitis. J Gastroenterol 2008, 43(6):473-483.

35. Zhou L, Tan JH, Cao RC, Xu J, Chen XM, Qi ZC, Zhou SY, Li SB, Mo QX, Li ZW et al: ATF6 regulates the development of chronic pancreatitis by inducing p53-mediated apoptosis. Cell Death Dis 2019, 10(9):662.

36. Li J, Ma X, Liu C, Li H, Zhuang J, Gao C, Zhou C, Liu L, Wang K, Sun C: Exploring the Mechanism of Danshen against Myelofibrosis by Network Pharmacology and Molecular Docking. Evid Based Complement Alternat Med 2018, 2018:8363295.

\section{Figures}




\section{Xiaochaihu Decoction}

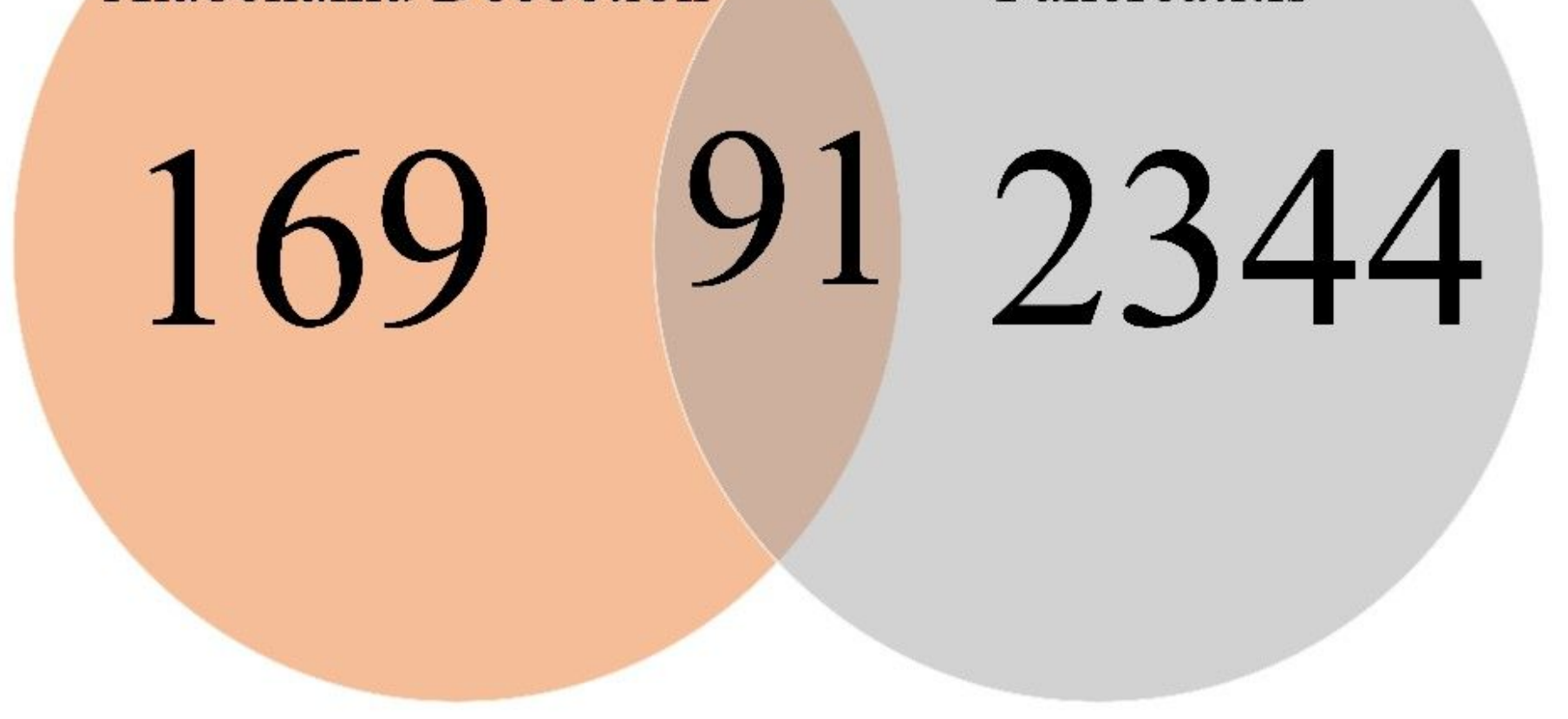

Figure 1

Venn map of common targets on Xiaochaihu Decoction and Pancreatitis. The orange circle indicated the targets of Xiaochaihu Decoction and the gray circle indicated the targets of pancreatitis. The part of two intersecting circles were the common targets. 


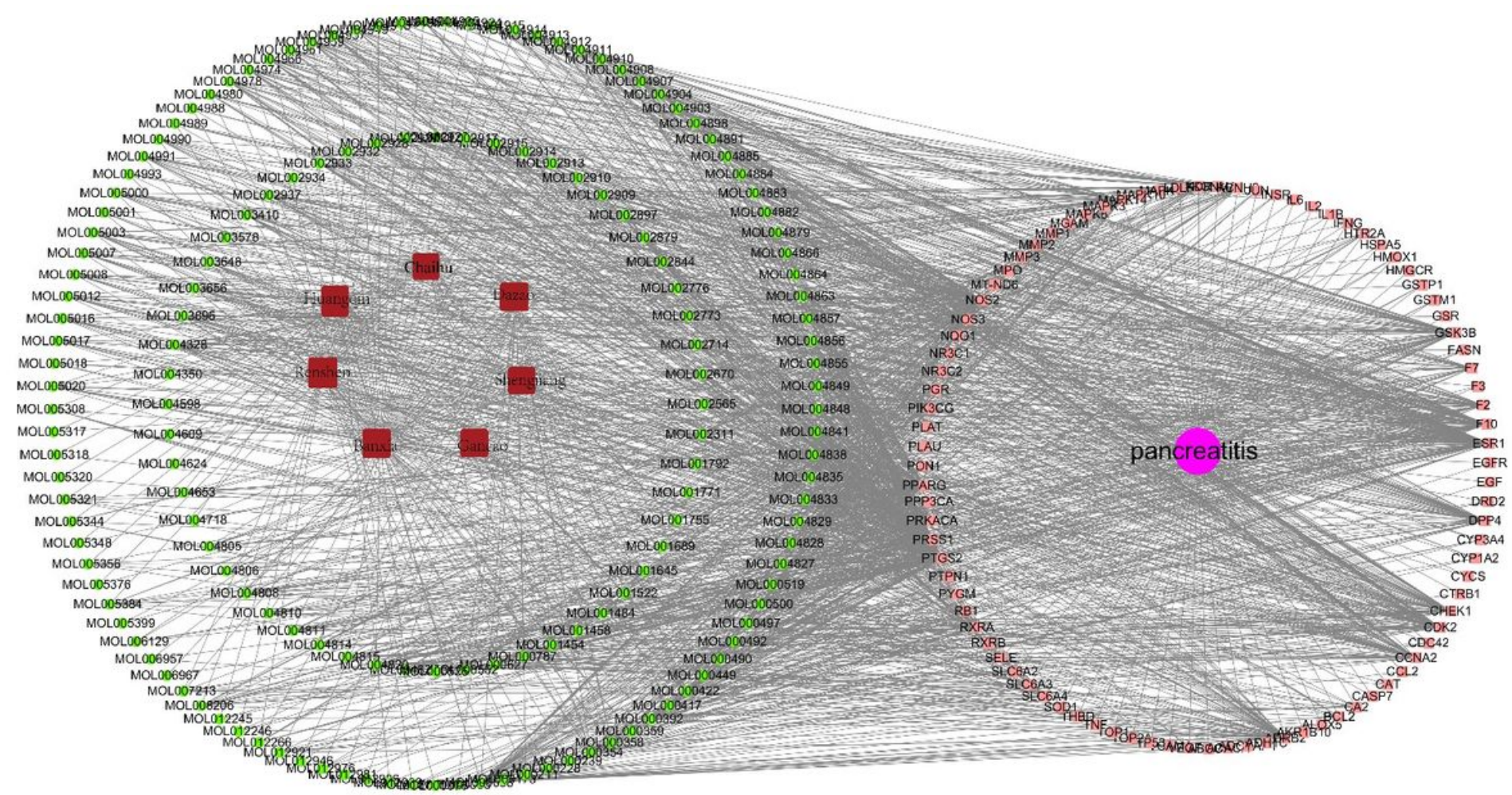

Figure 2

Herb-Compound-Target-Disease network. The red square indicated the herb name, the green circle indicated the compounds, the orange circle indicated the target and the pink circle indicated the disease name.

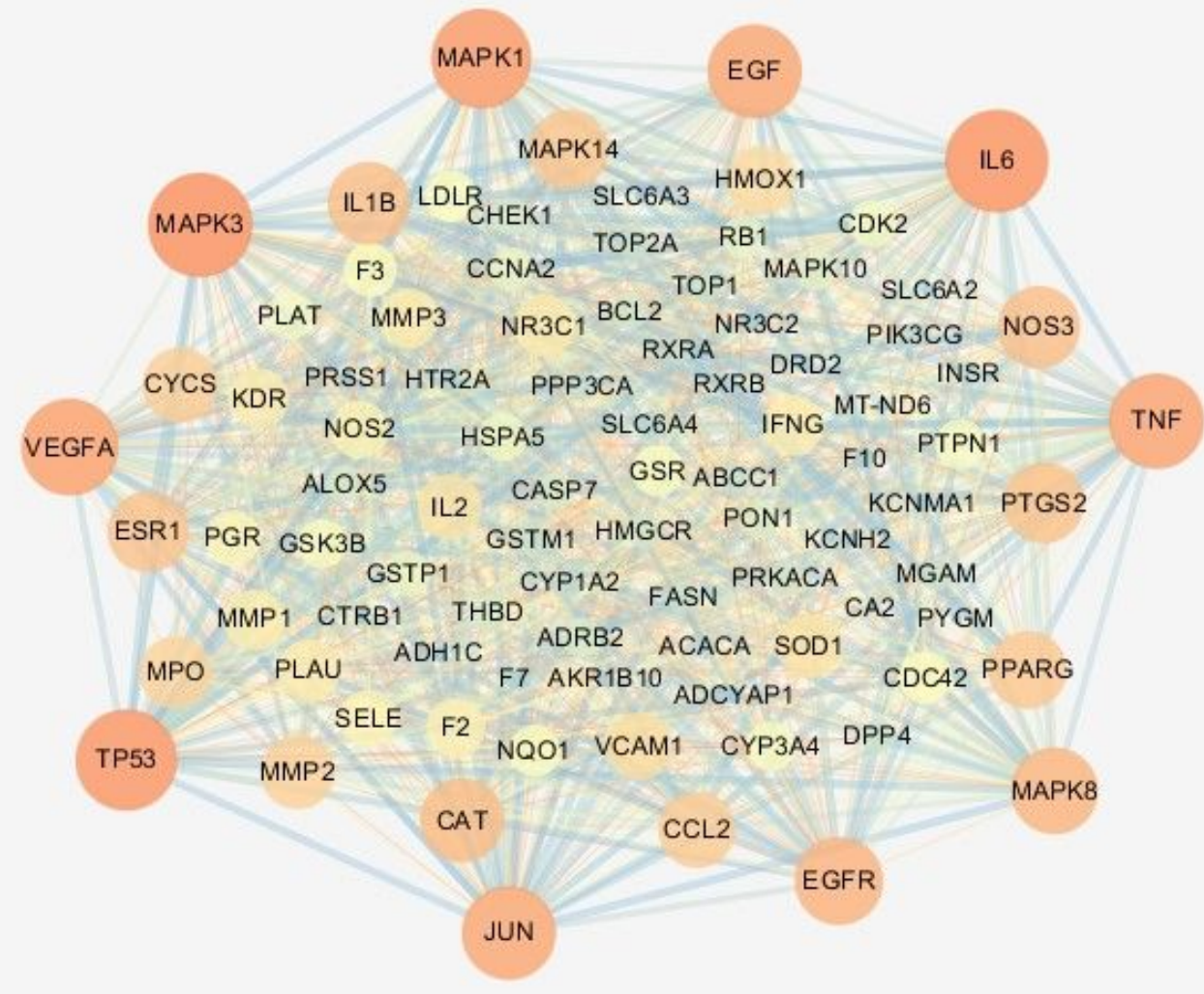


Figure 3

Protein - protein interaction network (PPI interaction network). Orange, yellow, green circles indicated the proteins. The lines represented the nodes associated through protein-protein interactions.

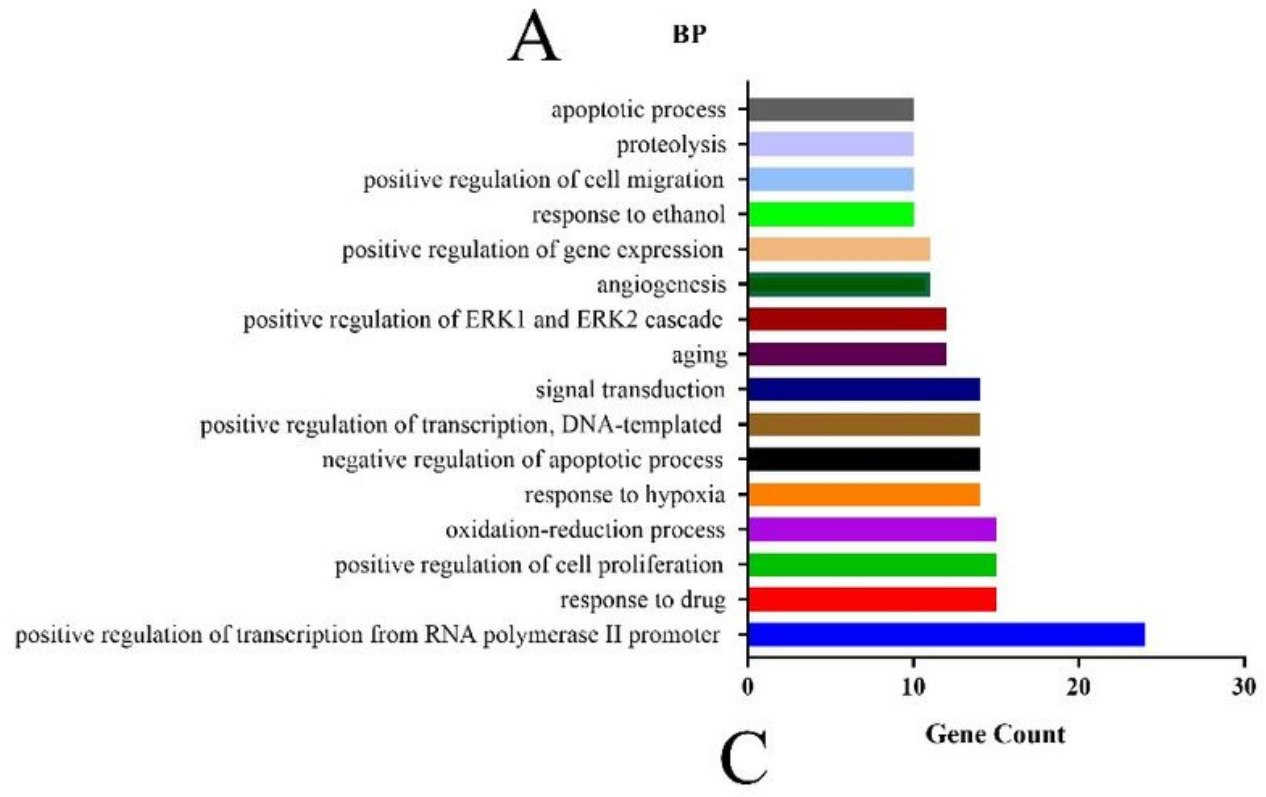

CC
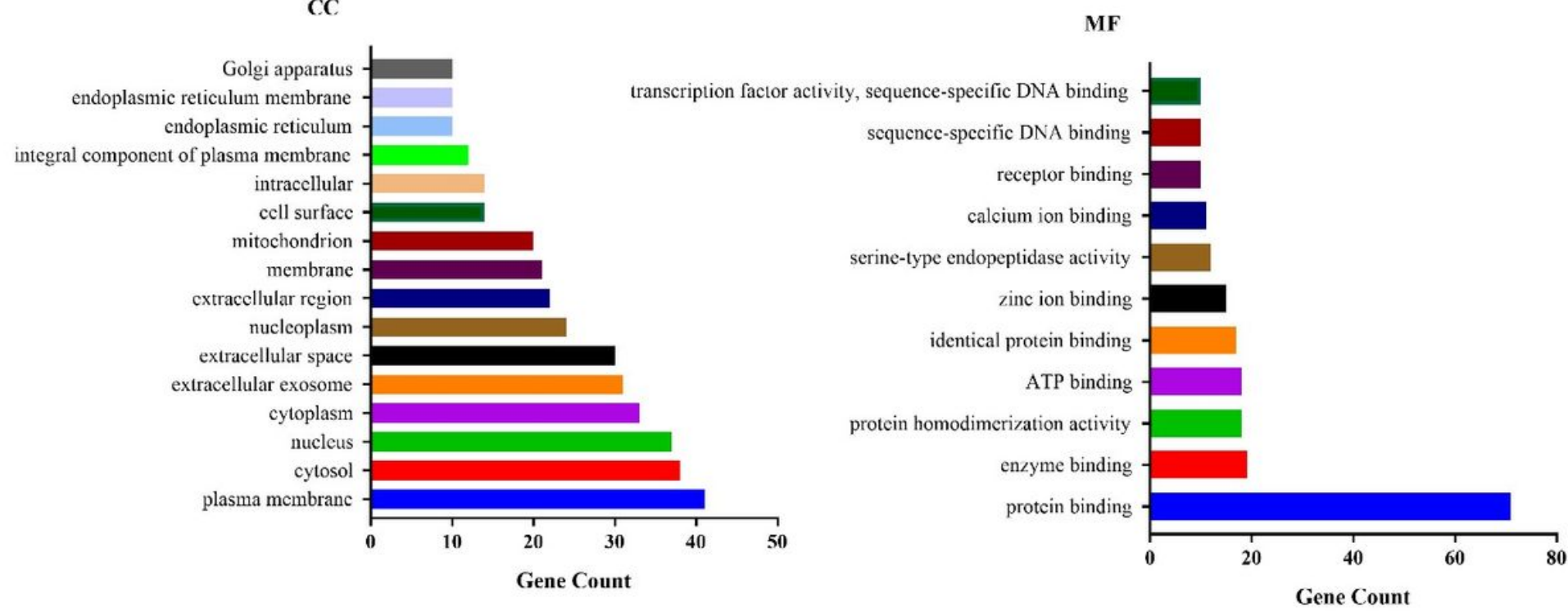

Figure 4

Gene Ontology (Go) enrichment analysis of the top 20 pathways. (A) Biological Process (BP). (B) Cell Component (CC). (C) Molecular Function (MF). 


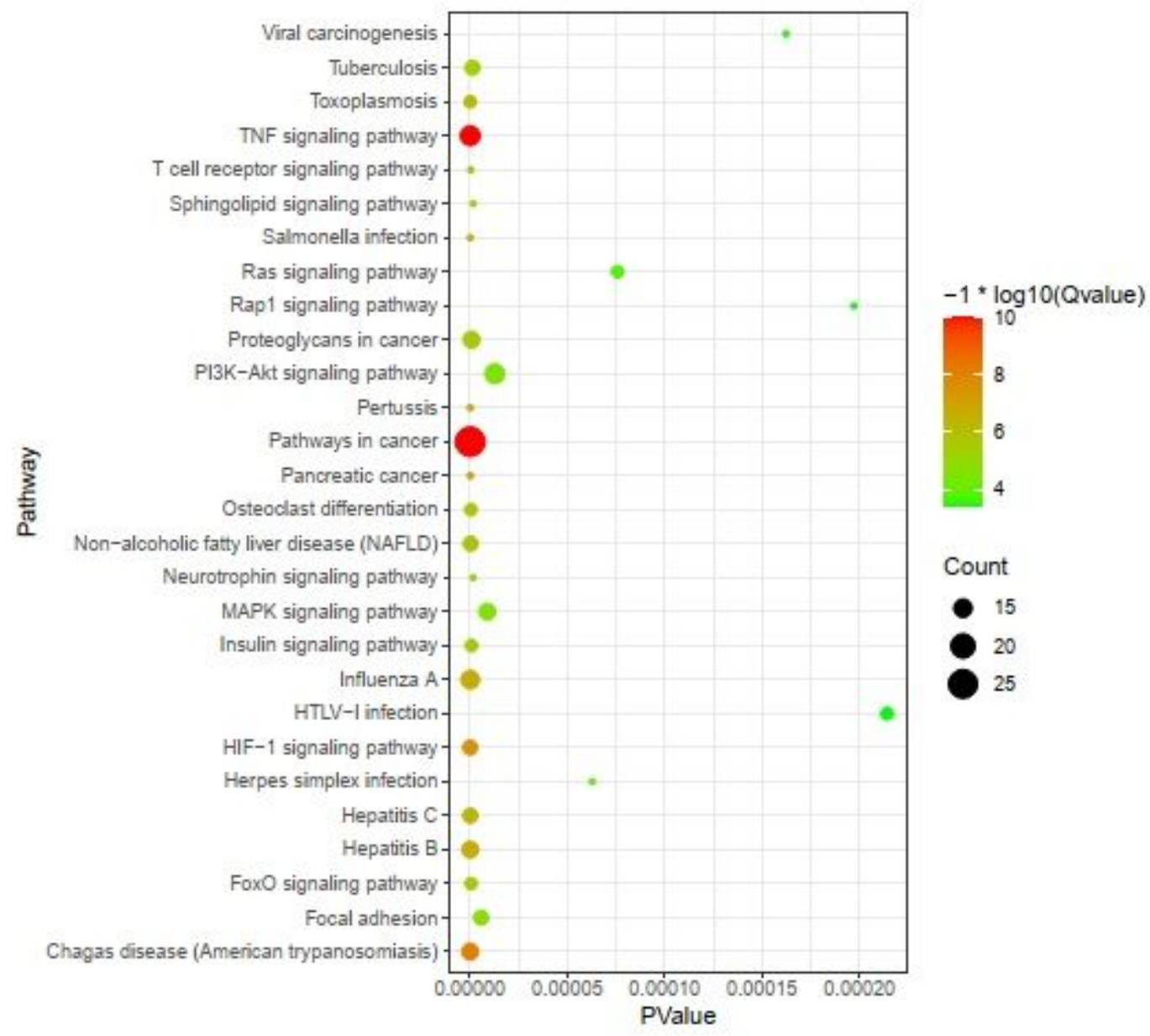

\section{Figure 5}

Kyoto Encyclopedia of Genes and Genomes (KEGG) pathway enrichment. The color of circles was represented the Qvalue and the size of circles was represented the Count. 
A

(2:0

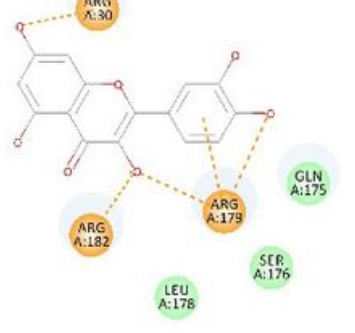

플
B

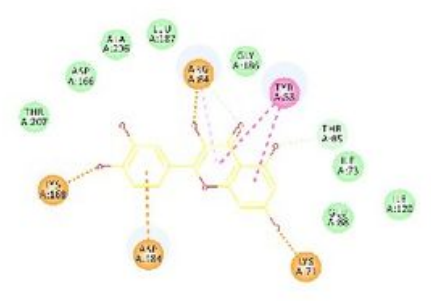

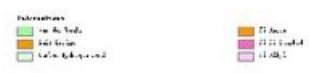

$\mathrm{D}$

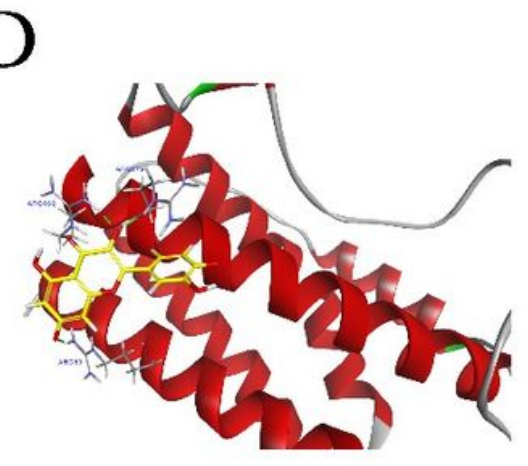

$\mathrm{E}$

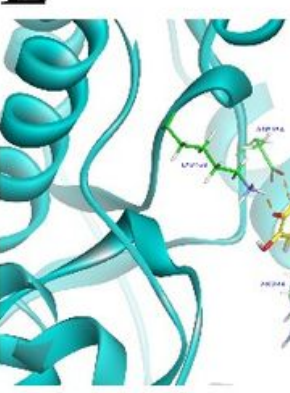

$\mathrm{C}$

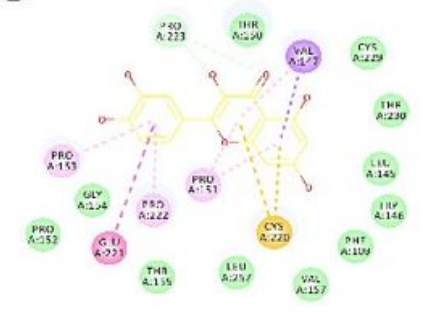

플

$\mathrm{F}$

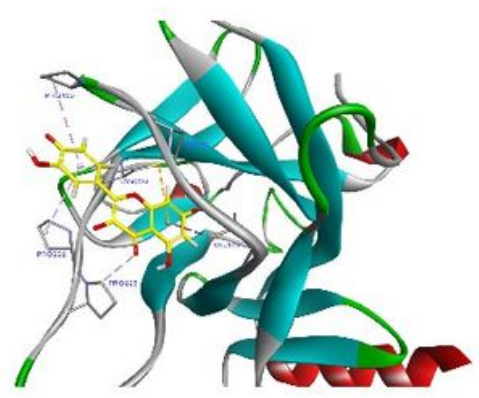

Figure 6

The protein-ligand of the docking simulation. (A) Quercetin and IL-6 (2D). (B) Quercetin and MAPK3 (2D). (C) Quercetin and TP53 (2D). (D) Quercetin and IL-6 (3D). (E) Quercetin and MAPK3 (3D). (F) Quercetin and TP53 (3D).
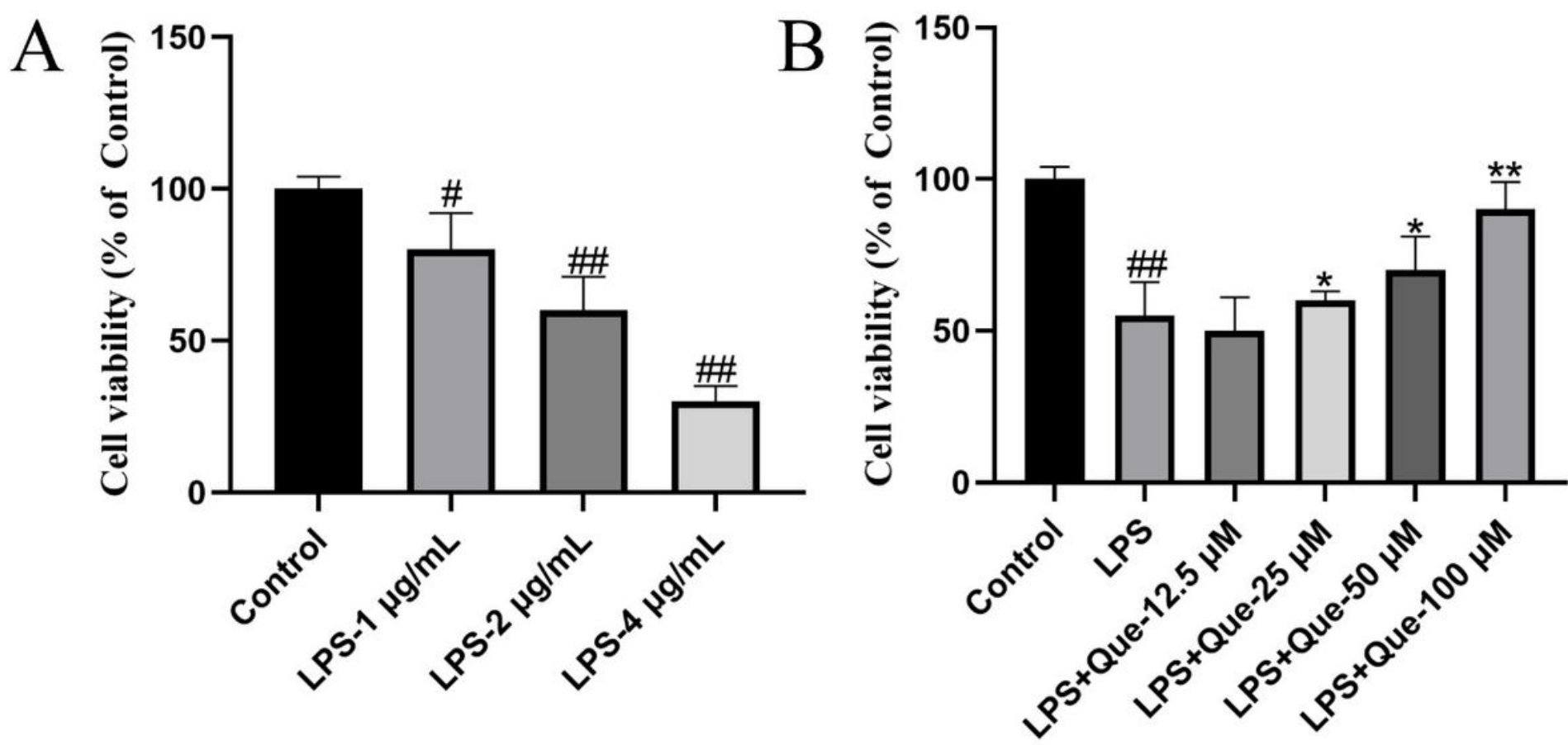
Figure 7

The effect of LPS and Quercetin at different dose on AR42J cells viability. (A) AR42J cells were treated with LPS at 1, 2, $4 \mu \mathrm{g} / \mathrm{mL}$ for 24 hours. (B) AR42J cells were incubated with Quercetin $(12.5,25,50,100$ $\mu \mathrm{M})$ for 8 hours after treated with $1 \mu \mathrm{g} / \mathrm{mL}$ LPS. Data are expressed as the mean \pm SD of three independent experiments. \#P<0.05, \#\#P<0.01 compare with the control group; ${ }^{*} P<0.05$ and ${ }^{*} \mathrm{P}<0.01$ compare with the LPS group.
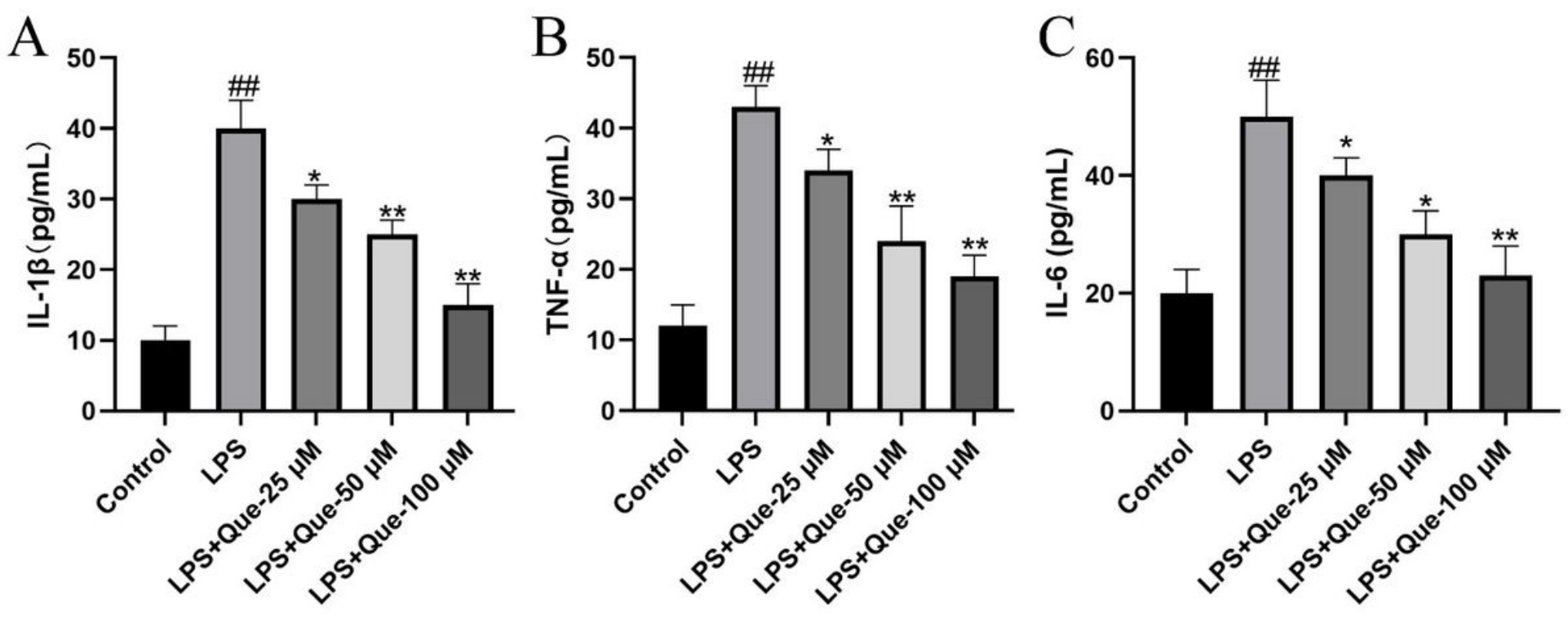

\section{Figure 8}

The effects of Quercetin on the production of IL-6, IL-1 $\beta$ and TNF- $a$ in LPS-induced pancreatitis in vitro. After LPS $(1 \mathrm{mg} / \mathrm{mL})$ treated with AR42J cells for 24 hours, Quercetin at dose of 25, 50, $100 \mu \mathrm{M}$ were incubated with the cells for another 8 hours. And the production of IL-1 $\beta$ (A), IL-6 (B), and TNF- $a(C)$ were detected by Elisa kit. Data are expressed as the mean $\pm S D$ of three independent experiments. $\# P<0.05$, \#\#P $<0.01$ compare with the control group; ${ }^{*} \mathrm{P}<0.05$ and ${ }^{*} \mathrm{P}<0.01$ compare with the LPS group. 
A
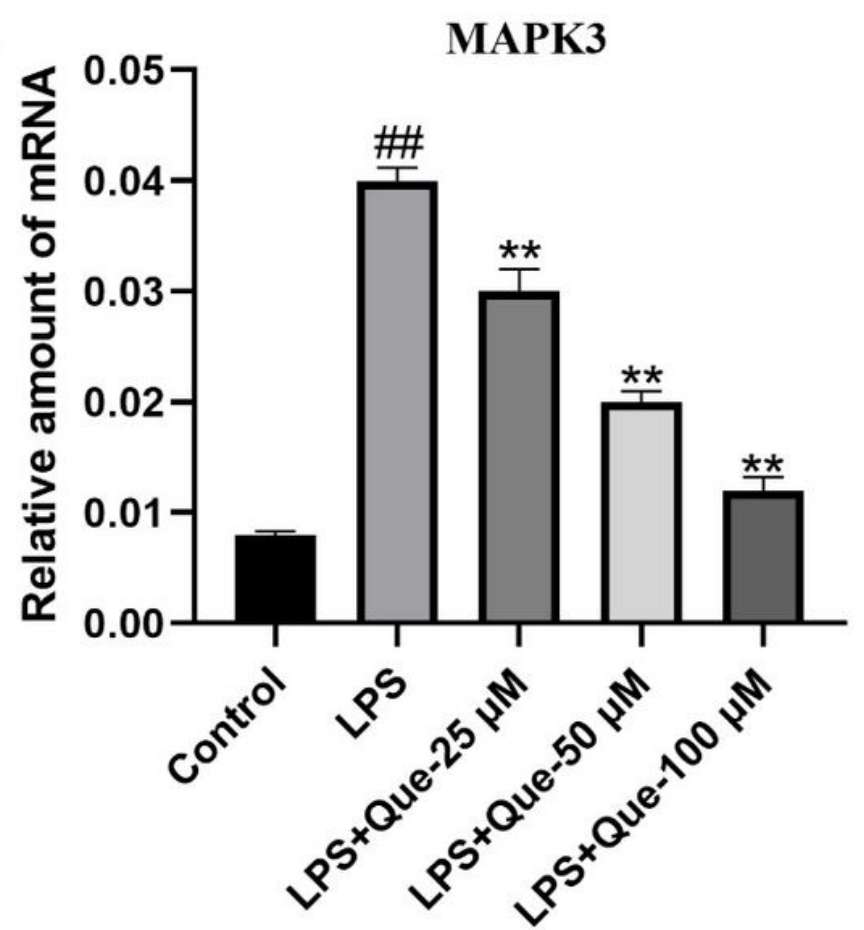

$\mathrm{B}$
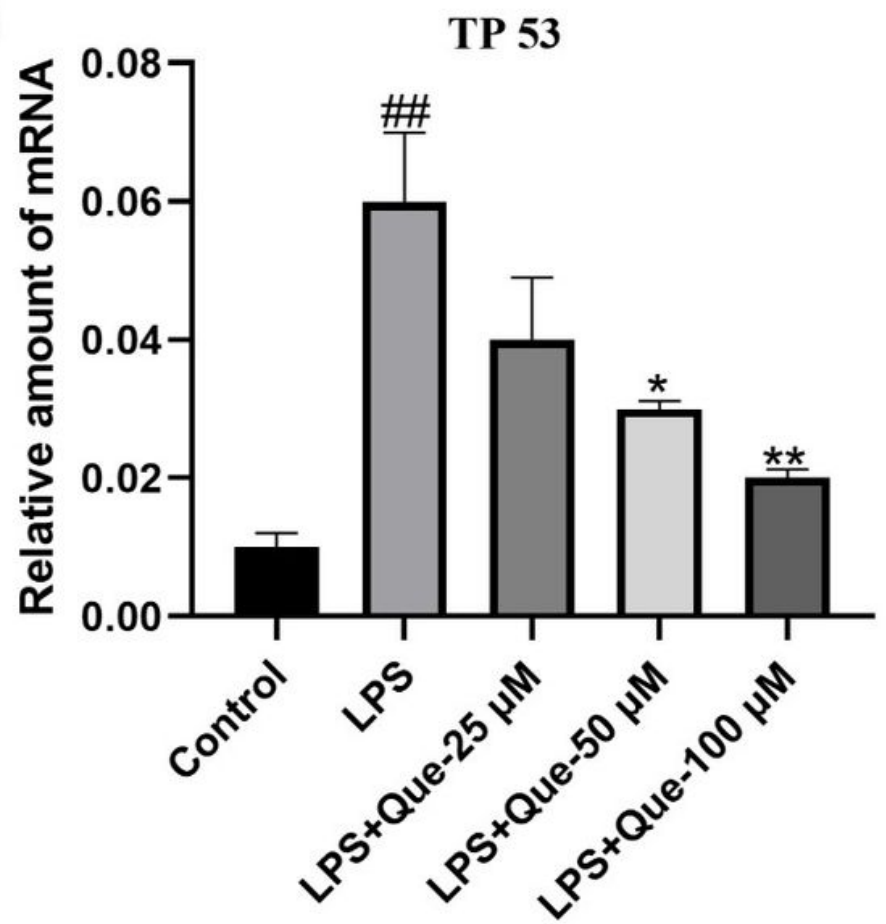

Figure 9

Changes in mRNA expressions of MAPK3 and TP53 in AR42J cells following LPS and (or) Quercetin treatment. (A) Real-time PCR analysis of MAPK3 in AR42J cells. (A) Real-time PCR analysis of TP53 in $A R 42 J$ cells. Data are expressed as the mean $\pm S D$ of three independent experiments. $\# P<0.05, \# \# P<0.01$ compare with the control group; ${ }^{*} \mathrm{P}<0.05$ and ${ }^{*} \mathrm{P}<0.01$ compare with the LPS group. 